\title{
Comportamiento del consumidor en páginas webs. Tipología de usuarios y respuesta visual ante la comunicación de marca
}

\section{Consumer Behavior on Web Pages. User Typology and Visual Response towards Brand Communication}

\author{
Leonardo Ortegón Cortázar (Colombia) \\ Institución Universitaria Politécnico Grancolombiano \\ lortegon@poli.edu.co
}

\begin{abstract}
Resumen
El estudio de la comunicación de marca es uno de los aspectos de gran relevancia para la gestión de mercadeo de las empresas. La tendencia en comunicación comercial se ha desplazado a todo tipo de entornos, en los que predominan, a manera de estudio, las plataformas y dispositivos digitales (Santomier, 2008), de modo que la gestión de marca se ve enfrentada a nuevos retos para capturar y
\end{abstract}

\begin{abstract}
The study of brand communication is one of the most relevant marketing aspects in companies. The tendency to have commercial communication has displaced all types of environments, where platforms and digital devices predominate as study (Santomier, 2008). This way, brand management faces new challenges to capture and keep the attention of consumers in order to produce favorable
\end{abstract}

$\begin{array}{lr}\text { FECHA DE RECEPCIÓN: } & 17 \text { de nobiembre de } 2011 \\ \text { FECHA DE REVISIÓN: } & 21 \text { de enero de } 2012 \\ \text { APROBACIÓN: } & 19 \text { de abril de } 2012\end{array}$
Para citar este artículo / to cite this article Ortegón Cortázar, L. Comportamiento del consumidor en páginas webs. Tipología de usuarios y respuesta visual ante la comunicación de marca. Poliantea 8 (14), 33-50. 
mantener la atención de sus consumidores a fin de producir preferencias y actitudes favorables hacia sus comunicaciones en línea. Este estudio presenta una revisión del comportamiento del consumidor en función de su tipología de uso y respuesta visual ante la comunicación de marca en páginas webs, en la que se articulan técnicas provenientes de neuromercadeo como Eye Tracker incluyendo la gráfica de calor (HeatMap) y registro de patrones visuales (Gazeplot). Se demostró la necesidad de integrar múltiples factores de diseño estético y funcional para especificar las relaciones e influencias sobre la compra virtual.

Palabras clave: mercadeo digital, página web, comunicación de marca, neuromercadeo. preferences and attitudes towards online communication. This study revises the consumer's behavior towards their use typology and visual answer with respect to brand communication on web pages, where techniques that come from neuromarketing, such as Eye Tracker, including heat graphic (HeatMap) and record of visual patterns (Gazeplot), are articulated. It was possible to show the need to integrate multiple factors of aesthetic and functional design to specify the relations and influences over a virtual purchase.

Keywords: Digital market, web page, brand communication, neuromarketing. 


\title{
Comportamiento del consumidor en páginas webs. Tipología de usuarios y respuesta visual ante la comunicación de marca
}

\author{
Leonardo Ortegón Cortázar (Colombia) \\ Magíster en Psicología del Consumidor. \\ Profesor universitario de Investigación de Mercados y Comportamiento del Consumidor.
}

Según Santomier (2008), la gestión y comunicación de las marcas a nivel global se está desarrollando por medio de múltiples plataformas y dispositivos digitales. Una de las formas más difundidas para lograr esta comunicación entre marcas y consumidores es la página web (sitio web) que promueven una empresa o una marca, la cual es considerada como la herramienta de publicidad interactiva más importante en la actualidad (Sicilia, Ruiz \& Munuera, 2005), pues se encarga de convertir el tráfico en usuarios o compradores, recibe los números y los transforma en valor tangible para el negocio (Ryan \& Jones, 2009; Vega, 2009). En este sentido, el cambio de medios de la comunicación de marca se convierte en uno de los tópicos de interés ya que tiene una relación directa con la lealtad de los consumidores (González, Orozco \& De la Paz, 2011) y con el valor de las ventas de las marcas (Ortegón, 2010, 2012). Desde el anterior punto de vista, en lo que se refiere al mercadeo digital, la página web de la empresa ocupa el primer lugar en importancia. Es la pieza vital hacia la cual todas las otras actividades en línea dirigen sus esfuerzos. En complemento, Ghose y Dou (1998) afirman que Internet representa un gran potencial para las comunicaciones de mercadeo en el futuro.

Según Klein (2003), el comercio web es reconocido también por el potencial de proveer información completa y actualizada de los consumidores, 
así como la creación de experiencias virtuales con los productos. La tendencia de comunicación de marca por medio del comercio web ha sido estudiada en diversos informes (Cyr, Kindra \& Dash, 2008), en los que se percibe un incremento del número de usuarios interconectados. Colombia no es ajena a esta tendencia (Mora \& Hurtado, 2011), y es así como en la actualidad se calcula que hay más de cinco millones de suscriptores a Internet (fijo o móvil), lo cual se traduce en más de 35 millones de personas que navegan por la red. Se subraya además que un crecimiento del diez por ciento en la penetración de Internet puede incrementar el crecimiento de un país en el uno por ciento (Valero, 2011). En complemento, Ryan y Jones (2009) señalan que, no solamente hay más personas conectadas, sino que además usan Internet más seguido, durante periodos más largos y realizan más actividades en la red; como resultado, la penetración del mercadeo por canales digitales se ha incrementado a gran velocidad en los últimos años.

Esta proliferación de nuevas tecnologías ha facilitado la revolución de las comunicaciones en la gestión de marca, con lo cual estas se enfrentan al reto de obtener, no solo la atención, sino la aprobación de sus consumidores para establecer una experiencia de comunicación, lo que de acuerdo con Vargo y Lusch (2004) ha generado una transición en la lógica del mercadeo de una visión centrada en los bienes tangibles a una nueva visión centrada en el valor intangible y las experiencias que la marca pueda construir en la mente de los consumidores, manifiestas en experiencias y comportamientos sensoriales de preferencia o evitación. Con esta nueva visión, Vargo y Lusch (2004) afirman que el valor debe ahora ser definido y cocreado con el consumidor, en lugar de ser "entregado" o "enviado" a este por parte de la empresa, por lo cual se deben buscar estrategias o métodos para recibir una retroalimentación de la comunicación de marca en páginas webs.

Estos resultados hacen evidente la necesidad de explorar la conducta del consumidor y su interacción con el comercio web. El consumo virtual, según Nielsen Online (2007), ha tenido un incremento sostenido en los últimos años, por lo que se puede afirmar que el comercio web representa una oportunidad para la industria y gestión de las marcas. Según 
Ranchhod (2004), los canales tradicionales de mercadeo están siendo disueltos o asimilados en una red global que es alimentada por este canal de comunicación, lo cual implica el desarrollo de estrategias de mercadeo "activas", en vez de "pasivas", desarrollar comunicaciones en las páginas webs que activen la atención del usuario y permita mantener procesos de exploración y navegación. Por tanto, este autor afirma entonces que la mejor utilización de las últimas tendencias tecnológicas se convierte en una tarea fundamental en mercadeo y los negocios, modifica la costumbre de la interacción física comprador/vendedor que tradicionalmente se daba. La información se puede acceder desde cualquier lugar y a cualquier hora, de manera que el mercadeo y la publicidad están en la necesidad de reinventarse para asegurar que son apropiadas a la nueva realidad tecnológica (Ozuem, Howell \& Lancaster, 2008).

Según Beck (2000), Internet también se ha convertido en uno de los canales principales para el mercadeo de bienes relacionados con la industria de la moda, puesto que provee tanto los medios para que el minorista presente sus últimas tendencias mediante fotografías, video y sonido, como el uso de una página web que le permite venderle directamente a sus consumidores (Hines \& Bruce, 2007). Según Interactive Media, los ingresos por ventas en línea de productos relacionados con la moda han tenido un crecimiento anual del veinticinco al treinta por ciento desde el 2000 (Hines \& Bruce, 2007). Sin embargo, el estudio de Kim y Stoel (2004) identificó que un gran número de estas marcas se limitaban a usar la página web como medio de información únicamente. Goldsmith y Flynn (2004) sugieren que los minoristas que están usando con mayor éxito los canales remotos son aquellos enfocados en la venta de productos estándar y no quienes venden productos de diseño a la medida.

De acuerdo con Ryan y Jones (2009), todo en una página web debe estar diseñado en función de los objetivos de conversión, ya sean directos (información de los productos o servicios, pedido de compras, formularios, boletines, etc.) $\mathrm{o}$ indirectos (información acerca de la marca o el negocio que genere confianza, contenido que repercuta en visitas repetidas, etc.). La conversión es la clave del éxito del mercadeo digital.

Según Ryan y Jones (2009), el punto central de referencia de una 
página web debe ser la página de inicio. Esta debe ofrecer un tipo de navegación intuitiva que dirija a los usuarios a las secciones principales, además de contar con una descripción adecuada del sitio que brinde una idea general del negocio a los posibles consumidores. Se recomienda que incluya, resaltados, los nuevos productos y servicios, ofertas especiales, incentivos, noticias y cualquier otro tipo de promoción (Ryan \& Jones, 2009).

\section{Usabilidad y accesibilidad en páginas webs}

La usabilidad y la accesibilidad se encargan de que el contenido del sitio pueda ser consultado por la mayor cantidad de audiencia posible y que la entrega de información a los usuarios se realice de forma que para ellos sea confortable y familiar (Ryan \& Jones, 2009).

En lo que se refiere a usabilidad, para que los usuarios consigan lo que buscan en Internet de manera efectiva se recomienda que el diseño sea simple, elegante y funcional. El objetivo es eliminar la frustración de la experiencia del usuario, asegurándose de eliminar las barreras, para completar sus objetivos sin esfuerzo (Ryan \& Jones, 2009).
El término accesibilidad hace referencia al proceso de diseñar una página web que sea alcanzable por todas las personas. Un buen diseño debería permitirles igual acceso a la información y funciones que provee el sitio. Para lograr esto existen parámetros que son aplicables a todo tipo de página web (Ryan \& Jones, 2009), los cuales garantizan que las ofertas comerciales sean igualmente asequibles (Anderson \& Srinivasan, 2003; Bhattacherjee, 2001). Los consumidores pueden comparar y contrastar los productos y servicios de la competencia con un mínimo gasto de tiempo y esfuerzo personal. Esta situación implica una alta competencia en precios y mínima lealtad, por lo que existe un creciente interés en la comprensión de las bases de la satisfacción y lealtad del cliente en el comercio web (Srinivasan, Anderson \& Ponnavoul, 2002).

La complejidad y orden visual son características de diseño que afectan la respuesta inicial hacia una página web (Lavie \& Tractinsky, 2004), con la cual se influencia la posterior experiencia del usuario con la página (Van der Heijden, 2003). Numerosos estudios muestran los efectos de estos factores en variables como facilidad percibida de uso, efectividad de la 
comunicación y satisfacción del usuario (Deng \& Poole, 2010).

Por otra parte, en la literatura específica sobre el comportamiento del consumidor a través de Internet se insiste en los factores personales y externos que influyen en la experiencia de exploración visual y la satisfacción para las intenciones de compra futuras (Bigné, Ruiz \& Andreu, 2004). Estas intenciones de compra por Internet también han sido explicadas por un modelo propuesto por Li, Kuo y Russel (1999), quienes describen que el comportamiento de compra en la red se ve influenciado por características sociodemográficas, conocimiento del medio o canal de comunicación y la experiencia de exploración visual proporcionada por el sitio web. En complemento a la anterior propuesta, Anderson y Srinivasan (2003) plantean otro modelo en el que se analiza el impacto de las características relativas al consumidor y a la empresa que pueden acentuar o reducir la influencia en la satisfacción y la lealtad con la página web. En particular, se centran en tres variables individuales (inercia, motivación de conveniencia y tamaño de compra), junto con dos variables afines a la empresa (confianza y valor percibido ofrecido por la compañía). En este orden de ideas, se presentan algunas características de tipología de consumidores relacionadas con la frecuencia de exploración y compra en el comercio web.

\section{Características asociadas a mayor frecuencia de exploración y compra en páginas webs}

Según Li, Kuo y Russel (1999) y Bigné, Ruiz y Andreu (2004), las características asociadas a una mayor frecuencia de exploración y compra en los entornos digitales están contenidas en las características sociodemográficas, la estrategia empresarial de la compañía en su página web y el nivel de satisfacción del consumidor en compras anteriores por Internet.

Entre las características sociodemográficas que facilitan la exploración, uso y compra por Internet están el nivel de formación, el sexo y el nivel de ingresos del usuario. Li, Kuo y Russel (1999) parten de la premisa de que el nivel de formación está positivamente relacionado con los ingresos y estos con el comportamiento de compra del consumidor en Internet. De este modo, dichos autores contrastan empíricamente que los compradores por medio de 
Internet tienen un nivel de formación más alto que aquellos usuarios que no realizan compras virtuales. Análogamente, Rodríguez, Meseguer, Hormigo y Gerard (1999) desarrollaron un trabajo que identifica el nivel de educación como una de las variables desencadenantes del desarrollo del comercio electrónico. Por lo tanto, el nivel de formación es una característica relevante a ser considerada. Con respecto a la característica sociodemográfica de sexo, Bigné, Ruiz y Andreu (2004) indican que existe una mayor proporción de compradores varones; no obstante, estos mismos autores señalan una tendencia creciente de la participación femenina en las páginas webs debido a una mayor exposición a la tecnología en el lugar de trabajo, aspecto especialmente significativo debido a que en los últimos años las mujeres han accedido a mayor cantidad de puestos cualificados en el mercado laboral (Modahl, 2000). Por otra parte, las motivaciones para utilizar la web por parte de hombres y mujeres también difieren y dan lugar a comportamientos de compra distintos (Dholakia \& Uusitalo, 2002). En esta línea, el estudio de Yoon, Cropp Cameron (2002) ofrece evidencia empírica de que, a pesar de utilizar las mujeres Internet como herramienta de búsqueda y realizar la compra en los establecimientos tradicionales, los hombres presentan mayor predisposición hacia las transacciones electrónicas. Con respecto al nivel de ingresos, Bigné, Ruiz y Andreu (2004) indican que cuanto mayor es este, mayor es su propensión marginal al consumo y, por tanto, aumenta la probabilidad de que adquiera los equipos necesarios para poder desarrollar sus compras por Internet. Para Donthu y García (1999) los hogares que más se exponen a los entornos digitales, en los que se incluyen páginas webs, tienen un nivel de ingresos superior al promedio de la población.

En cuanto a la estrategia empresarial de la compañía en su página web, en la actualidad el usuario que hace uso de la página tiene un perfil muy específico, por lo que no todos los productos son susceptibles de tener éxito en Internet (Peterson, Balasubramanian y Bronnenberg, 1997). Entre las principales características asociadas a un mayor uso y compra en los entornos digitales se encuentran las particulares de los productos ofertados que son valoradas por los consumidores, y la influencia de los 
precios en la compra. Sobre la gestión de producto, la Asociación de Investigación de los Medios de Comunicación, AIMC (2004) señala que los productos más demandados en Internet por parte de los consumidores son: pasajes de avión, computadores, entradas a espectáculos, libros, revistas, alojamiento, programas informáticos, música, aparatos electrónicos, películas y, en general, bienes cuyas características pueden evaluarse perfectamente con anterioridad a la compra. Según Peterson, Balasubramanian y Bronnenberg (1997), el nivel de uso y compra en las páginas webs depende del precio del producto, la frecuencia de compra, la tangibilidad y el grado de diferenciación del producto o servicio. En comparación, Girard, Silverblatt y Korgankoar (2002) clasifican la gestión de producto en tres categorías: búsqueda, experiencia e información, y concluyen que los bienes de búsqueda son los que más éxito tendrán en Internet ya que son aquellos en los que el riesgo percibido de compra por los consumidores es menor. Respecto a la influencia de los precios en el comportamiento de uso y compra en las páginas webs, Bailey (1998) señala que las características de Internet permiten la ejecución instantánea del pedido y la eliminación de los costos de intermediación, lo que posibilita un precio muy competitivo para el cliente final.

En cuanto al nivel de satisfacción del consumidor en compras anteriores por Internet y su relación con el uso y compra en las páginas webs, Bigné, Ruiz y Andreu (2004); Giese y Cote, 2000 y Oliver (1997) señalan que la satisfacción con el producto o servicio en sí, proporciona un resultado de agrado derivado del consumo. En consecuencia, cuando un consumidor está satisfecho por el desarrollo del servicio se espera que su comportamiento a corto plazo sea congruente con su satisfacción, en este caso, con el comportamiento de exploración, uso y compra por medio de la página web. Por otra parte, Kim y Stoel (2004) realizaron una investigación empírica para examinar los atributos que favorecen la satisfacción del consumidor. Las evaluaciones se basaron en la experiencia de mujeres con sus páginas de ropa favoritas. Los hallazgos indicaron que la información adecuada, la capacidad de transacciones y el tiempo de respuesta eran dimensiones que influenciaban la satisfacción del consumidor, mientras que la apariencia visual, los aspectos estéticos o de entretenimiento, no contribuían significativamente al bienestar 
del usuario. Sin embargo, en contradicción con Kim y Stoel (2004), el trabajo de Siddiqui, McColl y Birtwistle (2003) al examinar los sitios web de ropa, reportó que los consumidores buscaban experiencias únicas mediante Internet, en las cuales pudieran ver acercamientos de las prendas en $3 \mathrm{D}$, tener mayor interactividad $\mathrm{y}$ más emoción.

\section{Exploración de páginas webs. Comportamiento visual del consumidor con Eye Tracking}

De acuerdo con Usability.gov, una de las técnicas más apropiadas para evaluar el comportamiento del consumidor es el Eye Tracker (seguimiento de ojos). La tecnología de Eye Tracking ha sido usada en muchas áreas, como sistemas visuales, lenguaje cognitivo y diseño de productos. Es una herramienta muy útil para el diseño de entornos digitales debido a que muestra cuáles son las áreas de la página que capturan la atención del usuario y las que son ignoradas. Además sirve para conocer cómo los usuarios buscan la información que necesitan (O'Connell, 2009).

Los métodos tradicionales para representar el comportamiento de exploración y uso en las páginas webs son Heat Map y Gaze Plot. El primero representa las áreas de una página web en las que se concentran la mirada y la atención de los visitantes; se realiza con el uso de lentes y receptores especiales que vigilan el movimiento de los ojos y captan los puntos específicos de la pantalla donde la mirada recae más frecuentemente. Al realizar estos estudios con cientos de personas y en cientos de páginas ${ }^{1}$ se pueden descubrir patrones de comportamiento que indican las áreas en las cuales la mirada y las acciones de un usuario se fijan con mayor frecuencia. Esta información se puede graficar en forma de áreas en las que se marcan con colores diferenciados aquellas de intensidades mayores y menores, hasta formar algo parecido a un mapa con curvas de niveles que puede superponerse a la página web para así determinar cuáles son las áreas más visitadas. En la figura 1 se presenta un ejemplo de esta técnica de medición del comportamiento de exploración visual.

Los estudios con este método también señalan que los rostros de las personas tienen mayores niveles

\footnotetext{
Al respecto el doctor Philip Michel Zeman (2009) afirma que veinte personas generan resultados con una confiabilidad del 95\% con esta técnica de investigación.
} 
de atención, por lo que se diferencian con color rojo. En complemento a esta técnica, la investigación realizada por Nielsen (2006) muestra que los visitantes leen el contenido web en un patrón con forma de $\mathrm{F}$. Este patrón en $\mathrm{F}$ se refiere al orden de visualización: los usuarios comienzan por leer de un lado a otro la parte superior, ven hacia abajo, un poco hacia la derecha y luego bajan la mirada por el lado izquierdo.

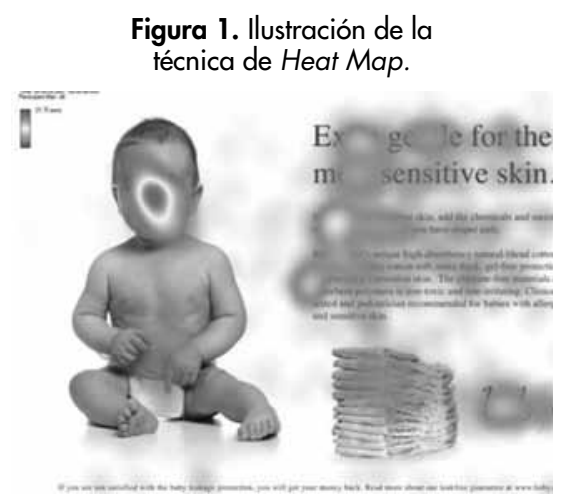

Fuente: http://www.neurosciencemarketing.com/blog/ articles/baby-heat-maps.htm.

Como se puede ver en la figura 2, existe una mayor fijación visual en la esquina superior izquierda de la página. Es claro que los usuarios no leen el contenido total de la página web; la información más importante debe encontrarse en los dos primeros párrafos (Nielsen, 2006).

En contraste, la técnica Gaze Plot señala el número y orden de las fijaciones realizadas sobre una página web. Señala la ruta visual que traza el visitante al explorar una página. En la figura 3 se ilustra esta técnica mediante círculos en los que, a mayor tiempo de fijación, se incrementa su tamaño; cada círculo se encuentra enumerado en forma secuencial de acuerdo con el recorrido y la atención visual de la página.

Figura 2. Patrón en $\mathrm{F}$ generado por Eye Tracking.

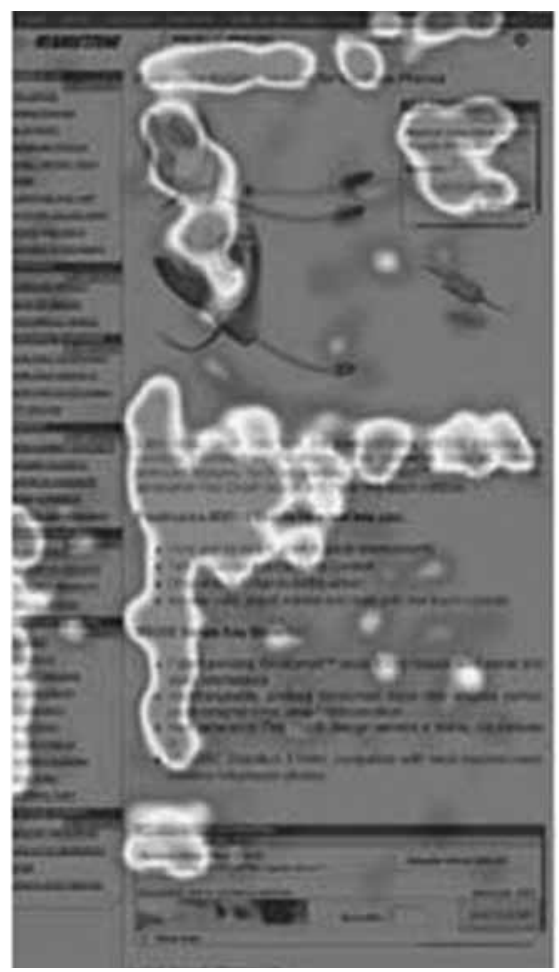

Fuente: Nielsen (2006). 
Figura 3. llustración de la técnica Gaze Plot.

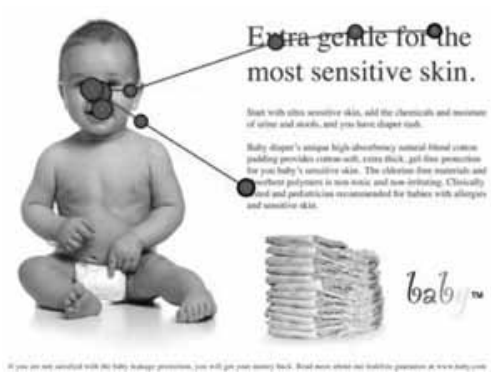

Fuente: http://www.neurosciencemarketing.com/blog/ articles/baby-heat-maps.htm.

En la figura 3 se observa la ruta de exploración visual de un consumidor que inicia su recorrido en la mitad de la página, se desplaza hacia la cabeza del niño y finaliza en el título de la pieza publicitaria.

Estas dos formas de medición de la conducta visual provenientes del neuromercadeo, son relativamente recientes en el escenario de la investigación del consumidor, por lo que se proyectan como herramientas poderosas para ayudar a comprender de una forma alternativa el mismo comportamiento ante la comunicación de marca en páginas webs.

En años recientes el neuromercadeo se ha venido consolidando como una de las herramientas de investigación más innovadorasy controvertidas en el campo del comportamiento del consumidor. El principal argumento a favor del uso del neuromercadeo en la investigación del consumidor consiste, de acuerdo con Fleming (2006), en el hallazgo proveniente de las neurociencias con respecto a la dificultad o imposibilidad por parte de los consumidores de expresar las razones emocionales que generan sus hábitos de consumo y sus reacciones a los distintos estímulos de mercadeo, en este caso la comunicación de marca en páginas webs. En otras palabras, para los consumidores resulta muy difícil expresar verbalmente el nivel de activación o vínculo emocional que pueden llegar a experimentar con una marca en el comercio web. Además, de acuerdo con este autor, los resultados de varias investigaciones recientes apuntan al hecho de que las decisiones que toman los consumidores se basan principalmente en sensaciones subjetivas, de carácter emocional, y estas sensaciones están vinculadas con estímulos sensoriales que se activan en el momento del consumo, en este caso, la exploración, uso y compra en una página web.

\section{Discusión}

Dadas las coyunturas actuales en lo referente a medios de comunicación, gracias a la web 2.0 y a Internet de alta velocidad, los consumidores 
tienen más control que nunca. Cada vez con más frecuencia, estos desean la oportunidad de estar involucrados en el diseño de los productos, para así expresar más efectivamente su imagen, convirtiéndose en "prosumidores". Pueden elegir el contenido que deseen, en la forma que lo prefieren (Ryan \& Jones, 2009). De acuerdo con Chang, Chen y Huang (2009), la participación directa del usuario lleva a una mayor satisfacción durante el consumo. Este hecho también se relaciona con una gran revolución en los hábitos de compra, lo que a su vez hará que se modifique por completo la relación existente entre el consumidor y los establecimientos comerciales tradicionales (Alba, Lynch, Weitz, Janiszewski, Lutz, Sawyer \& Wood, 1997; Sharma \& Sheth, 2004). Lynch, Kent y Srinivasan (2001) sostienen que el impacto del diseño de la web en la lealtad del consumidor y en las intenciones de compra futura depende de las características de los productos ofertados; en consecuencia, las investigaciones previas reconocen la necesidad de estudiar los factores que ejercen una influencia en la satisfacción y compra del consumidor por medio de Internet. No obstante, la diversidad de factores plantea la necesidad de analizarlos de manera integrada y, de este modo, especificar las relaciones de las influencias sobre la compra virtual (Bigné, Ruiz \& Andreu, 2004). Según Deng y Poole (2010), estas características de diseño visual de la página deben contar también con interfaces que promuevan atracción y placer, en comparación a ser solo funcionales, puesto que hoy en día a los consumidores se les presenta mayor cantidad de opciones ante las cuales los usuarios pueden navegar de una página a otra sin mayor esfuerzo.

Finalmente, para próximos trabajos es necesario exponer algunas sugerencias metodológicas con el fin de enriquecer el estudio en este tema. Se recomienda complementar las técnicas y resultados aportados por el método del Eye Tracker con entrevistas en profundidad que indaguen experiencias y actitudes de los usuarios cuando son expuestos a páginas webs, esto debido a que las técnicas expuestas provenientes del neuromercadeo relacionan la conducta visual que ocurre durante la exposición en la comunicación de marca de la página, pero no logran indagar las creencias, pensamientos y actitudes frente a ella o específicamente al tipo de la(s) marca(s), 
conductas de compra futuras. Sería interesante también correlacionar estos resultados referentes al comportamiento visual del consumidor y sus respuestas verbales a otro tipo de entornos digitales con el fin de mejorar la comprensión de sus niveles de satisfacción e intenciones de compras futuras.

\section{Referencias}

Alba, J., Lynch, J., Weitz, B., Janiszewski, C., Lutz, R., Sawyer, A. \& Wood, S. (1997, julio). Interactive Home Shopping: Consumer, Retailer and Manufacturer Incentives to Participate en Electronic Marketplaces. Journal of Marketing, 61, 38-53.

Anderson, R. E. \& Srinivasan, S.S. (2003). E-Satisfaction and E-Loyalty: A Contingency Framework, Psychology \& Marketing, 20, (2), 123-138.

Bailey, J. (1998). Intermediation and electronic markets: aggregation and pricing in internet commerce. Cambridge: Massachusets Institute of Technology.

Beck, B. (2000). Key strategic issues in online apparel retailing: The Need For An Online Fitting Solution. Recuperado de http://www.techexchange.com/thelibrary/.
Bigné, Ruiz \& Andreu (2004). Conceptualización del comportamiento del consumidor en los entornos virtuales. Un análisis cualitativo. Revista de estudios sobre el consumo, 70, 9-20.

Chang, C.C., Chen, H.Y. \& Huang, I.C. (2009). The Interplay between Customer Participation and Difficulty of Design Examples in the Online Designing Process and Its Effect on Customer Satisfaction: Mediational Analyses. Cyberpsichology \& Behavior, 12, (2), 147-154.

Cyr, D., Kindra, G. \& Dash, S. (2008). Web site design, trust, satisfaction and e-loyalty: the Indian experience. Online Information Review, 32, (6), 773-790.

Deng, L. \& Poole, M.S. (2010). Affect in web interfaces: A study of the impacts of web page visual complexity and order. MIS Quarterly, $34,(4), 711-730$.

Dholakia, R. \& Uusitalo, O. (2002). Switching to Electronic Stores: Consumer Characteristics and the Perception of Shopping Benefits. International Journal of Retail Distribution and Management, 30, (10), 459-469.

Donthu, N. \& García, A. (1999, mayo-junio). The internet shopper. 
Journal of Advertising Research, 39, (3), 52-58.

Estudio General de Medios-Colombia. (2011, febrero). Estudio general de medios EGM: perfil demográfico del usuario de Internet. $P \mho M, 54$.

Fleming, J. (2006). Is that a neuromarketer in your brain? Gallup Management Journal. Princeton, NJ, EE. UU.: The Gallup Organization.

Ghose, S. \& Dou, W. (1998). Internet functions and their impacts on the appeal of internet presence sites. Journal of Advertising Research, 38, (2), 29-43.

Giese, J.L. \& Cote, J.A. (2000). Defining Consumer Satisfaction. Academy of Marketing Science Review (online), 2000 (01). Recuperado de http://www.amsreview.org/articles/ giese01-2000.pdf

Girard, T., Silverblatt, R. \& Korgankoar, P. (2002). Influence of Product Class on Preference for Shopping on the Internet. Journal of Computer Mediated Communications, 8, (1). Recuperado de http://www.ascusc.org/jcmc/vol8/issuel/girard. html.

Goldsmith, R. \& Flynn, L. (2004). Psychological and behavioural drivers of online clothing purchase. Journal of Fashion Marketing and Management, 8 , (1), 84-95.

González, E., Orozco, M.M., De la Paz, A. (2011). El valor de la marca desde la perspectiva del consumidor. Estudio empírico sobre preferencia, lealtad y experiencia de marca en procesos de alto y bajo involucramiento de compra. Revista de Contaduría y Administración, 235, 217-240.

Hines, T. \& Bruce, M. (2007). Fashion Marketing: Contemporary Issues (2a Ed.). Oxford: Elsevier.

Kim, S. \& Stoel, L. (2004). Apparel retailers: website quality dimensions and satisfaction. Journal of Retailing and Consumer Services, 11, (2), 109-117.

Klein, L.R. (2003). Creating virtual product experiences: The role of telepresence. Journal of Interactive Marketing, 17, (1), 41-55.

Lavie, T. \& Tractinsky, N. (2004). Assessing Dimensions of Perceived Visual Aesthetics of Web Sites. International Journal of Human-Computer Studies, 60, (3), 269-298.

Li, H., Kuo, C. \& Russell, M. (1999). The Impact of perceived Channel 
Utilities, Shopping Orientations, and demographics on the Consumer's Online Buying Behaviour. Recuperado de http://www.ascusc.org/ jcmc/vol5/issue2/hairong.html.

Lynch, P.D., Kent, R.J., Srinivasan, S.S. (2001). The global Internet shopper: evidence from shopping tasks in twelve countries. Journal of Advertising Research, 41, (3), 15-23.

Modahl, M. (2000). Now or never. Nueva York: Harper Collins.

Mora, M. \& Hurtado, C. (2011). ¿Cuáles son las percepciones, usos $y$ hábitos de los colombianos sobre las tecnologías de la información y las comunicaciones? Recuperado el 20 de marzo de 2011, en http://www.mintic.gov.co/news. asp? articleId $=237$.

Nielsen Online (2007). Eighty one million people in the U.S. watch broadband video at home or work, according to Nielsen and CTAM. Recuperado de http://www.nielsen. com/media/2007/pr_070717.html.

Nielsen, J. (2006). F-Shaped Pattern For Reading Web Content. Recuperado el 15 de marzo de 2011, en http://www.useit.com/alertbox/reading_pattern.html.
O'Connell, C. (2009). Eyetracking and Web site Design. Recuperado el 15 de marzo de 2011, en http:// www.usability.gov/articles/newsletter/.

Oliver, R.L. (1997). Satisfaction. A Behavioral Perspective on the Consumer. Singapur: McGraw-Hill.

Ortegón, L. (2010). Panorama actual de los métodos de medición de marcas y valor de marca desde la orientación al consumidor. Poliantea, 11, 170-187.

(2012). Exploración del valor de marca y su correlación con el valor de las ventas. Un estudio aplicado en compañías agroquímicas. Ciencias Estratégicas, 20, 27.

Ozuem, W., Howell, K. \& Lancaster, G. (2008). Communicating in the new interactive marketspace. European Journal of Marketing, 42, (9/10), 1059-1083.

Peterson, R.; Balasubramanian, S.; \& Bronnenberg, B. (1997). Exploring the Implications on the Internet for Consumer Marketing. Journal of the Academy of Marketing Science, 25, (4), 329-346. 
Ranchhod, A. (2004). The changing nature of cyber-marketing strategies. Business Process Management Journal, 10, (3), 262-276.

Rodríguez, I., Meseguer, A., Hormigo, E. \& Gerard, R. (1999). El futuro de la venta telemática en España a través de la world wide web: un modelo estructural. IX Encuentro de Profesores Universitarios de Marketing. Madrid: ESIC.

Ryan, D. \& Jones, C. (2009). Understanding Digital Marketing: Marketing strategies for engaging the digital generation. Londres, Inglaterra: Kogan Page.

Santomier, J. (2008, octubre). New media, branding and global sports sponsorship. International Journal of Sports Marketing and Sponsorship 10, (1).

Sharma, A. \& Sheth, J. (2004). Web based marketing. The coming revolution in marketing thought and strategy. Journal of Business Research, 57, 696-702.

Sicilia, M., Ruiz, S. \& Munuera, J.L. (2005). Effects of interactivity in a Web site. Journal of Advertising, 34, (3), 31-45.
Siddiqui, N., Antonia, O., McColl, J. \& Birtwistle, G. (2003). Retailer and consumer perceptions of online fashion retailers: Web site design issues. Journal of Fashion Marketing and Management, 7, (4), 345-355.

Srinivasan, S.S., Anderson, R. \& Ponnavoul, K. (2002). Customer Loyalty in E-commerce: An Exploration of its Antecedents and Consequences. Journal of Retailing, 78, 41-20.

Then, N. \& Delong, M. (1999). Apparel shopping on the web. Journal of Family and Consumer Sciences, 91, (3), 65-68.

Valero, D. (2011). Internet pasaría a ser un derecho de todo ciudadano en Colombia. Recuperado el 28 de agosto de 2011, de El Tiempo.com: http:// www.eltiempo.com/politica/internetpasaria-a-ser-un-derecho-de-todociudadano_10239829-4.

Van der Heijden, H. (2003). Factors Influencing the Usage of Websites: The Case of a Generic Portal in The Netherlands. Information and Management, 40, 541-549.

Vargo, S.L. \& Lusch, R.F. (2004). Evolving to a new dominant logic for marketing. Journal of Marketing, $68,(1), 1-17$. 
Vega, R. (2009). Internet y el mer- Zeman, P.M. (2009). How to Get the cadeo farmacéutico: el cambio de Best Eye-Tracking Results for Your la relación laboratorio-médico-pa- Website. Recuperado el 20 de marciente. Poliantea, 9, 26-38. zo de 2011, en http://www.stepfor-

Yoon, D., Cropp, F. \& Cameron, G. (2002). Building relationships with th.com/blog/2009/getting-best-eyetracking-results-for-your-website/. portal users: the interplay of motivation and relational factors. Journal of Interactive Advertising, 3, (1). Recuperado de http://jiad.org/article25. 